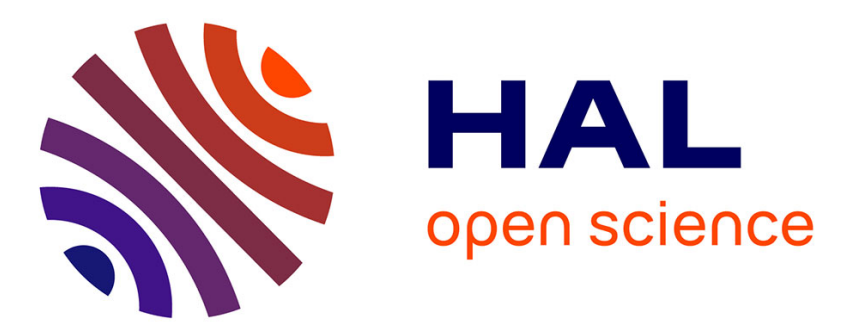

\title{
Balance control for an underactuated leg exoskeleton based on capture point concept and human balance strategies
}

C. Bidard, Christine Chevallereau, Vaiyee Huynh

\section{- To cite this version:}

C. Bidard, Christine Chevallereau, Vaiyee Huynh. Balance control for an underactuated leg exoskeleton based on capture point concept and human balance strategies. 2016 IEEE-RAS 16th International Conference on Humanoid Robots (Humanoids), Nov 2016, Cancun, Mexico. pp.483-488, 10.1109/HUMANOIDS.2016.7803319 . cea-01840419

\section{HAL Id: cea-01840419 https://hal-cea.archives-ouvertes.fr/cea-01840419}

Submitted on 6 Dec 2019

HAL is a multi-disciplinary open access archive for the deposit and dissemination of scientific research documents, whether they are published or not. The documents may come from teaching and research institutions in France or abroad, or from public or private research centers.
L'archive ouverte pluridisciplinaire HAL, est destinée au dépôt et à la diffusion de documents scientifiques de niveau recherche, publiés ou non, émanant des établissements d'enseignement et de recherche français ou étrangers, des laboratoires publics ou privés. 


\title{
Balance Control for an Underactuated Leg Exoskeleton based on Capture Point Concept and Human Balance Strategies
}

\author{
Vaiyee Huynh $^{1,2}$, Catherine Bidard ${ }^{1}$ and Chistine Chevallereau ${ }^{2}$
}

\begin{abstract}
This paper presents a balance control for an active lower limbs exoskeleton based on the instantaneous capture point concept and human balance strategies. Our goal is to implement it on a real underactuated exoskeleton EMY. The control, inspired from biomechanic studies, aims at assisting an able-bodied person while preserving his comfort and his safety to the maximum. We first present briefly how the machine can balance itself according to disturbances which can come from the operator, and how it can imitate human balance strategies. We then show how we tackle the reduction of the number of actuators problem which leads to an underactuation/overactuation problem.
\end{abstract}

\section{INTRODUCTION}

In recent years, we are witnessing a growing interest in developping exoskeletons. An exoskeleton is a mechanical device, active or passive, more or less anthropomorphic, that operates in parallel with the human. The idea is that the operator wears the device like a suit in order to benefit from its abilities such as its power or its strength. In this paper, we will focus on powered lower limb exoskeletons. There are two main kinds of exoskeletons [1][2] : those developped to assist gait in rehabilitation and those developped to enhance the operator's performance. Some exoskeletons are like biped robots that carry the person inside in order to give them back some mobilities and eye contact with people around them, and some others are built for gait therapy. Performanceaugmenting exoskeletons [3][4], where the operator is well and able, have been developped for military domain, mostly load carrying and then, their applications are extended to industry. Studies showed for the early devices that although the load is mainly borne by the machine, the energetic consumption of the wearer increases, he requires more efforts to compensate for gait interference. However, in 2014, Mooney and al. [5] present a design of an autonomous leg exoskeleton that is capable of reducing the metabolic cost of walking with load. The challenge of building better leg exoskeletons is huge whatever the application.

To ensure the comfort and the safety of the operator, for both kinds of devices, balance control is essential for the coupled system \{operator + exoskeleton\}. Here, "balance" means that the system does not fall down and the feet are stationed. When using exoskeleton for mobility impaired person, the balance of the entire system is managed by crutches

\footnotetext{
${ }^{1}$ Vaiyee Huynh and Catherine Bidard are with CEA, LIST, Interactive Robotics Laboratory, F-91191 Gif-sur-Yvette, FRANCE vaiyee. huynh@cea. fr and catherine.bidard@cea.fr

${ }^{2}$ Vaiyee Huynh and Christine Chevallereau are with Institut de Recherche en Communications et Cybernetique de Nantes, Nantes, FRANCE christine. chevallereau@irccyn. ecnantes.fr
}

(Rewalk or eLegs), by the exoskeleton alone (REX) or by an external structure for gait rehabilitation (Lokomat). On the other hand, for able-bodied operator (Hv3 exoskeleton, BLEEX), the horizontal plane balance is assumed by the operator himself, since the device carries its own weight and an additional load. Balance control is an important field in biped robotics since falling down can cause severe damages for humanoid robots. In many studies, the system is described as simple models such as the Linear Inverted Pendulum (LIP) model [6] or Linear Inverted Pendulum Plus Flywheel model [7] or multi-body model [8]. Based on these, several stability conditions are established, implying center of mass (COM) placement for static and quasi-static balance, zeromoment point (ZMP) placement [9][10][11] or capture point placement [12][13], for dynamic balance.

In our works, we will consider load carrying exoskeletons which has to assist the operator, especially in balance recovery. Before assisting the human, we need to well understand his balance recovery strategies so that the exoskeleton reaction is compatible with the operator's. Therefore, we take inspiration from biomechanic works [14][15][16] to design a balance control for an active leg exoskeleton that reacts to external perturbations (possibly coming from the operator).

This paper presents briefly, in a first section, the balance control based on instantaneous capture point (ICP) [12][13][17] and human balance recovery strategies [14][15]. In [18], we presented simulation results of the balance control on a fully-actuated exoskeleton model. Because the real exoskeleton is underactuated (here, "underactuated" means all joints are not motorized), we study in a second section, the feasibility of the desired wrenches by the actuators in order to fulfill the desired tasks. So, we first analyze the system of one leg because the output of the balance control is a wrench that each leg should produce. Then, we quickly realize that one leg is an underactuated system and it is not possible to produce the desired wrench. Therefore, we analyze the system of two legs and finally we present different methods of torque computation and we compare their simulation results in section III. The paper finishes by a conclusion in section IV.

\section{BALANCE CONTROL}

The idea of this balance control is to assist a well and able person and to imitate human balance recovery mechanims in order to support the operator's action and reduce his effort while moving [18] : it has to preserve the entire system balance in any situations. 
Thanks to the tranparence of the machine [21], the exoskeleton is driven by the operator via a force-based (or torque-based) controller. We want to add a correction to this mode, produced by the exoskeleton, that preverves system balance and this task has to be realized by adding another force controller not to disturb the control principle. This is why the output of the balance control will be a desired wrench.

\section{A. Human Balance Strategies}

While using an exoskeleton, the operator is physically fastened to the device. To avoid producing opposite efforts to the human and therefore, destabilizing him, we need to understand how we manage our balance as humans. Some biomechanic researchers show that it exists three important mechanisms of human balance recovery [14][15][16] :

1) COM placement : the vertical projection of COM on the ground must remain inside the support polygon shaped by the feet.

2) Load/Unload mechanism : the weight that each leg should support is modulated according to the perturbation.

3) Stepping : we modify our support polygon in order to replace the COM inside (mechanism 1).

On flat floor, it has been showed that the regulation of angular momentum has a limited role, therefore we choose to neglect it [19].

Our objective is to design the balance control that respects these mechanisms as much as possible so that the exoskeleton acts in accordance to the operator's reaction. In this work, we consider an active exoskeleton standing on a flat ground and we suppose the exoskeleton transparent, it means that it can be driven easily by the operator. Moreover, we will only focus on stance leg(s) control : we let the operator to lead the swing leg movements if a step is taken to stay balanced. Later, we can imagine to extend our balance control by controlling the swing leg : [20] shows that we can match the operator natural step and the exoskeleton step still based on ICP.

\section{B. Balance Control for an Active Leg Exoskeleton}

We choose to describe the global behavior of the system with the LIP model [6] and to base our control on the instantaneous capture point (ICP) in order to have a smooth control on COM thanks to its dynamics [7][17][22][23]. Indeed, many studies like [13][20] show that human mecanisms, particularly in push recovery, seem to follow it. ICP gives an information on COM position and the direction in which it is moving (figure 1(a)) :

$$
\mathbf{X}_{I C P}=\mathbf{X}_{G C O M}+\frac{\dot{\mathbf{X}}_{G C O M}}{\omega_{0}}
$$

with $\omega_{0}=\sqrt{\frac{g}{z_{C O M}}}$ where $\mathbf{X}_{G C O M}=\left[\begin{array}{lll}x_{C O M} & y_{C O M} & 0\end{array}\right]^{T}$ is the Cartesian position of the vertical projection of COM, $\dot{\mathbf{X}}_{G C O M}=\left[\begin{array}{lll}\dot{x}_{C O M} & \dot{y}_{C O M} & 0\end{array}\right]^{T}$ is the Cartesian velocity of (a)

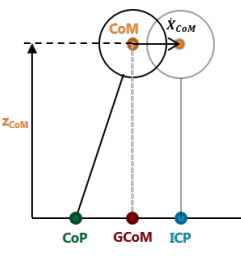

(b)

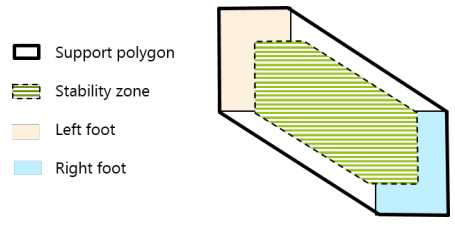

Fig. 1. (a) LIP model : Definition of ICP. (b) Stability zone.

GCOM, $g$ is the gravitational acceleration and $z_{C O M}$ is the height of COM.

First, we detect a loss of balance situation by observing ICP position in the support polygon and then, following the situation, we will correct its balance by controlling ICP position (mecanisms 1 and 3). Not to constrain the operator when the system is considered as stable, we define an area called stable zone which is a subpolygon of support polygon (figure 1(b)) and where no balance correction is necessary (the weight of the machine is still compensated). And for more stability facing low disturbances, we determine the contribution of each leg (mecanism 2) to generate joint torques to control the robot.

1) Instantaneous Capture Point Placement: The more ICP is close to the polygon's boundaries, the more the situation is unstable. Therefore, the first step of balance assistance is to correct ICP position, consequently COM position, if necessary. LIP model's dynamics show that placing judiciously the center of pressure (COP) of the system - the weighted average point of all the pressures over the contact area feet/ground - permits ICP to converge toward a desired position.

With a first order control law for the ICP defined by :

$$
\dot{\mathbf{X}}_{I C P}=K_{I C P} \cdot\left(\mathbf{X}_{I C P}^{(d)}-\mathbf{X}_{I C P}\right)
$$

we obtain the following COP expression via the equations of motion :

$$
\mathbf{X}_{C O P}=\mathbf{X}_{I C P}^{(d)}-\frac{K_{I C P}}{\omega_{0}} \cdot\left(\mathbf{X}_{I C P}^{(d)}-\mathbf{X}_{I C P}\right)
$$

where $\mathbf{X}_{C O P}, \mathbf{X}_{I C P}$ and $\mathbf{X}_{I C P}^{(d)}$ are the Cartesian positions of COP, ICP and desired ICP respectively, $K_{I C P}$ is the proportional gain and $K_{I C P}>0$ in order that ICP converges toward $\mathrm{ICP}^{(d)}$. COP must be inside the support polygon in other case, the feet will rotate : COP can't physically be outside. If the result of equation (3) leads to a COP outside of the stability zone, we use a heuristic to project it on the support polygon.

Thanks to the equations of motion and knowing COP and COM positions, we can calculate the produced acceleration at COM and then the wrench that the balance control needs to create the desired motion at COM :

$$
\mathbf{W}_{\text {corr }, C O M}=\left[\begin{array}{c}
\mathbf{F}_{\text {corr }} \\
\mathbf{0}
\end{array}\right]=\left[\begin{array}{c}
\frac{m g}{z_{C O M}}\left(\mathbf{X}_{G C O M}-\mathbf{X}_{C O P}\right) \\
\mathbf{0}
\end{array}\right]
$$

where $m$ is the mass of the system. 
We now know the entire effort that the actuators need to produce, let's :

$$
\mathbf{W}_{\text {act }, C O M}=\mathbf{W}_{\text {corr }, C O M}-\left(\mathbf{W}_{\text {weight }, C O M}+\mathbf{W}_{\text {dyn }, C O M}\right)
$$

where $\mathbf{W}_{\text {weight,COM }}$ is the wrench of gravity force applied to COM for weight compensation and $\mathbf{W}_{d y n, C O M}$ is the dynamic wrench. $\mathbf{W}_{\text {corr }, \text { COM }}$ represents the assistive wrench that helps the operator to recover from unstable situation.

2) Leg distribution: To calculate joint torques, we adopt the intuitive strategy of splitting $\mathbf{W}_{\text {act,COM }}$ into the two legs. Let's $\left(\alpha_{l} ; \alpha_{r}\right)$ left and right coefficients describing the contribution of each leg, with $\alpha_{l}+\alpha_{r}=1 . \alpha_{(l / r)}=0$ means that the associated leg is the swing one. Same strategy is proposed in [4][23][24] but the coefficients, based on ratio of distances, are not taking into consideration disturbances.

Depending on the feet configuration and the disturbance, the operator distributes his efforts in different ways [14]. We propose new coefficients $\alpha_{(l / r)}$ that take into account ICP position in the support polygon :

- compared to the feet $\left(\beta_{(l / r)}\right)$ : the more ICP is close to a foot, the more the contribution of the corresponding leg increases to 1 .

- compared to the support polygon limits $(\gamma)$ : when ICP gets closer to the limits, it means that the situation becomes unstable : we have to anticipate the step if ICP keeps moving and leaves the support polygon. The control has to help the operator to transfer the entire load of the machine from one leg to the other when needed by increasing the future support leg contribution to 1 .

Then, we build $\alpha_{(l / r)}$ that fulfill these criteria as :

$$
\alpha_{(l / r)}=f\left(\beta_{(l / r)}, \gamma\right)
$$

Finally, we obtain the wrench $\mathbf{W}_{a c t,(l / r)}$ that each leg should exert on its environment to realize the tasks :

$$
\mathbf{W}_{a c t,(l / r)}=\alpha_{(l / r)} \cdot \mathbf{W}_{a c t, C O M}
$$

3) Checking sole-ground contacts: Here, we check if the wrench computed by the control does not lead to a loss of contact. For that, we use the linearize version of Coulomb law :

$$
\mathbf{A}_{\mathbf{c}} \cdot \mathbf{F}_{\mathbf{c}}>b_{c}
$$

where $\mathbf{A}_{\mathbf{c}}$ describes linearized friction cone, $\mathbf{F}_{\mathbf{c}}$ is the contact force and $b_{c}$ is the friction coefficient. To simplify the problem, we neglect the dynamic part of $\mathbf{W}_{a c t, C O M}$. So, we assimilate $\mathbf{F}_{\mathbf{c}}$ to $\mathbf{W}_{a c t,(l / r)}$ written at the foot COP and in pratice, we calculate the ratio between horizontal components (correction forces) and the vertical component (weight compensation) as the moments are insignificant.

For a fully-actuated exoskeleton, we simply compute the joint torques using the Jacobian matrix. However, in reality, all joints of the exoskeleton are not motorized. Depending of the number of actuators on the machine, we can facing either an underactuated system or an overactuated system.

\section{UNDERACTUATED EXOSKELETON}

This section is dedicated to the computation of joint torques for an underactuated exoskeleton. Here, we determine the feasibility of the desired wrench that we previously determined. To lighten the exoskeleton, designers decide to reduce the number of actuators. However, the machine can no longer be able to produce all desired forces : are we able to create the desired wrench exactly ? What choices do we have ?

\section{A. EMY-Balance Exoskeleton}

EMY-Balance is a lower limbs exoskeleton designed and built by the Interactive Robotic Laboratory of CEA-LIST, for experimenting balance. It weighs about $36 \mathrm{~kg}$ without battery (42 kg with battery) and measures $1 \mathrm{~m} 62$. -Balance features 7 degrees of freedom per leg. Only 4 of them are actuated : two at the hip, one at the knee and one at the ankle.

\begin{tabular}{|c|c|c|}
\hline Joint & Motion & State \\
\hline Hip & Abduction/adduction & Active \\
\hline & Flexion/extension & Active \\
\hline & Rotation & Passive \\
\hline Knee & Flexion/extension & Active \\
\hline Ankle & Flexion/extension & Active \\
\hline & Inversion/eversion & Passive \\
\hline Sole & Flexion/extension & Passive \\
\hline
\end{tabular}

TABLE I

EMY-BALANCE'S DEGREES OF FREEDOM

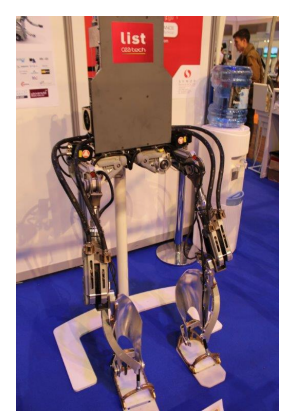

Fig. 2. EMY-Balance exposed at INNOROBO 2016 exhibition (ParisFRANCE)

\section{B. Issue}

Dealing with such a robot can actually imply two different cases :

- the system is underactuated : the exoskeleton can have difficulties to produce the computed wrench of the balance control.

- the system is overactuated or redundant : it can generate important internal forces.

The output of the balance control is a desired wrench for each leg that includes a weight compensation part and 
a balance correction part. When we analyze data, we will consider these two part separately :

- the weight compensation part : this part is always here because the device must carry its own weight. Therefore, the residual wrench which is not produced, is compensated by the operator and his energy consumption increases.

- the correction part : it is important that the exoskeleton is able to produce this part because the desired wrench is computed to assist the operator to recover from unbalance situations, else balance can be lost.

We suppose here that the human action is complementary to the robot action. So all the forces that we can not generate will be compensated by the operator.

1) System of one leg: First, we consider the system one leg because the output of the balance control is a desired wrench that each leg should produce in order to compensate a fraction of the weight and eventually to correct the balance of the system.

To simplify the problem, we do not consider the sole joint : the feet are flat and a leg has 4 active joints and 2 passive. First, we find the map matrix $\mathbf{L}_{a}$ that links active joint torques $\boldsymbol{\tau}_{a}$ to the wrench $\mathbf{W}$. If $\operatorname{rank}\left(\mathbf{L}_{a}\right)<6$, the system is underactuated. Else, $\operatorname{rank}\left(\mathbf{L}_{a}\right) \geq 6$, it realizes $\mathbf{W}$.

Let's write the COM twist in COM frame in function of active joints $\dot{\mathbf{q}}_{a}$ and passive joints $\dot{\mathbf{q}}_{p}$ :

$$
\mathbf{T}_{C O M}=\left[\begin{array}{c}
\mathbf{V}(G) \\
\boldsymbol{\omega}
\end{array}\right]=\mathbf{J}_{a} \dot{\mathbf{q}}_{a}+\mathbf{J}_{p} \dot{\mathbf{q}}_{p}
$$

where

- $\mathbf{V}(G)$ is the Cartesian COM velocity

- $\boldsymbol{\omega}$ is the angular COM frame velocity

- $\mathbf{J}_{a}$ is the Jacobian matrix of active joints only

- $\mathbf{J}_{p}$ is the Jacobian matrix of passive joints only

Then, the wrench we want to produce at COM is :

$$
\mathbf{W}=\left[\begin{array}{c}
\mathbf{F} \\
\mathbf{M}(G)
\end{array}\right]
$$

On one hand, with equations (9) and (10), we can write the mechanical power as :

$$
\mathbf{W}^{T} \mathbf{T}=\mathbf{W}^{T}\left(\mathbf{J}_{a} \dot{\mathbf{q}}_{a}+\mathbf{J}_{p} \dot{\mathbf{q}}_{p}\right)
$$

On the other hand, actuators power is given by :

$$
\tau_{a}^{T} \dot{\mathbf{q}}_{a}+\tau_{p}^{T} \dot{\mathbf{q}}_{p}
$$

Using (11) and (12) and virtual works principle, we obtain :

$$
\begin{aligned}
\boldsymbol{\tau}_{a} & =\mathbf{J}_{a}^{T} \mathbf{W} \\
\boldsymbol{\tau}_{p} & =\mathbf{J}_{p}^{T} \mathbf{W}
\end{aligned}
$$

Since $\boldsymbol{\tau}_{p}=\mathbf{0}$, we can create $\mathbf{W}$, if and only if :

$$
\mathbf{J}_{p}^{t} \mathbf{W}=\mathbf{O}_{2 \times 1}
$$

because $\mathbf{W}$ do not work with passive joints.
We can rewrite the equations as following :

$$
\mathbf{J}^{-t}\left(\begin{array}{c}
\mathbf{I}_{4} \\
\mathbf{O}_{2 \times 4}
\end{array}\right) \tau_{a}=\mathbf{W}
$$

where $\mathbf{J}=\left[\begin{array}{ll}\mathbf{J}_{a} & \mathbf{J}_{p}\end{array}\right]$ is nonsingular and invertible.

We note :

$$
\mathbf{W}=\mathbf{L}_{a} \boldsymbol{\tau}_{a}
$$

with $\mathbf{L}_{a}=\mathbf{J}^{-t}\left(\begin{array}{c}\mathbf{I}_{4} \\ \mathbf{O}_{2 \times 4}\end{array}\right)$. With 4 actuators per leg in EMYBalance, $\mathbf{W}$ belongs to a 4-dimension subspace and the components of $\mathbf{W}$ which do not verify (15) can not be generated by the leg.

Here, $\operatorname{rank}\left(\mathbf{L}_{a}\right) \leq 4$, the system of one leg is underactuated : we are not able to produce the desired wrench just considering one leg.

2) System of two legs: Since one leg does not fulfill its role, we combine the action of both legs. Let's :

$$
\mathbf{W}=\left[\begin{array}{ll}
\mathbf{L}_{a, l} & \mathbf{L}_{a, r}
\end{array}\right]\left[\begin{array}{c}
\boldsymbol{\tau}_{a, l} \\
\boldsymbol{\tau}_{a, r}
\end{array}\right]=\mathbf{L}_{a} \boldsymbol{\tau}_{a}
$$

Here, the whole system features 8 active joints for 4 passive joints and generally $\operatorname{rank}\left(\mathbf{L}_{a}\right)=6$ : combining both legs allows us to produce any wrench we want with a 2 degrees of redundancy that we can exploit. However, in this problem, although we could completely control the ICP, we could not freely choose the leg ditribution. And when the system is overactuated, we need to watch over the internal forces between the two legs, in order not to loose sole-ground contacts via sliding.

3) Methods of torques computation: We choose to take the whole system (two legs) to compute joint torques that fulfill the desired wrench. And the objective here, is to simulate and compare the different solutions we have before implementing the control in the real machine.

a) "Projection method": This method is used in [4] in 2003, for the exoskeleton BLEEX. The joint torques are computed as following : first, we consider the system as fully-actuated and we simply calculate joint torques via the Jacobian transposed matrix and then, at passive joints, we replace the prior torque value by zero :

$$
\boldsymbol{\tau}=\left(\begin{array}{c}
\boldsymbol{\tau}_{a} \\
\boldsymbol{\tau}_{p}
\end{array}\right)=\left(\begin{array}{c}
\boldsymbol{\tau}_{a} \\
\mathbf{O}_{2 \times 1}
\end{array}\right)
$$

All the efforts that are not produced by cancelling torques at passive joints, will be compensated by the operator.

b) "Optimization via the pseudoinverse matrix method": We want to produce a wrench as close as possible to the desired one, $\mathbf{W}_{\text {act,COM. }}$. We want :

$$
\mathbf{W}_{a c t, C O M}=\mathbf{W}_{a}=\mathbf{L}_{a} \boldsymbol{\tau}_{a}
$$


The shape of $\mathbf{L}_{a}$ is $6 \times 8$. We compute the torque vector with the minimal norm realizing the desired wrench :

$$
\boldsymbol{\tau}_{a}=\mathbf{L}_{a}^{+} \mathbf{W}_{a c t, C O M}
$$

with $\mathbf{L}_{a}^{+}=\mathbf{L}_{a}^{t}\left(\mathbf{L}_{a} \mathbf{L}_{a}^{t}\right)^{-1}$, the pseudoinverse matrix of $\mathbf{L}_{a}$.

This method guarantees the fulfilment of the desired wrench but does not take into consideration the desired leg distribution that ensures a better stability.

c) "Optimization via the pseudoinverse matrix under leg distribution constraint method": We exploit the 2dimension redundancy to add constraint to the problem. We use the singular value decomposition (SVD) to determine a kernel base of $\mathbf{L}_{a}$ :

$$
\mathbf{L}_{a}=\mathbf{U S V} \mathbf{V}^{t}=\mathbf{U}\left[\operatorname{diag}(\mathbf{S}) \quad \mathbf{O}_{6 \times 2}\right]\left[\begin{array}{l}
\mathbf{V}_{1}^{t} \\
\mathbf{V}_{2}^{t}
\end{array}\right]
$$

where $\mathbf{U}$ is an orthogonal matrix $6 \times 6$, $\mathbf{S}$ is a matrix $6 \times 8$ and $\mathbf{V}$ is an orthogonal matrix $8 \times 8$. $\mathbf{V}$ is composed of two parts : $\mathbf{V}_{1}$ which is a $8 \times 6$ matrix and $\mathbf{V}_{2}$ which is a $8 \times 2$. The columns of $\mathbf{V}_{2}$ constitute a kernel base of $\mathbf{L}_{a}$.

The set of solutions of (20) is :

$$
\begin{aligned}
& \boldsymbol{\tau}_{a}=\left[\begin{array}{c}
\boldsymbol{\tau}_{a, l} \\
\boldsymbol{\tau}_{a, r}
\end{array}\right]=\mathbf{L}_{a}^{+} \mathbf{W}_{a c t, C O M}+\mathbf{V}_{2} \mathbf{z} \\
& \boldsymbol{\tau}_{a}=\tilde{\boldsymbol{\tau}}_{a}+\left[\begin{array}{l}
\mathbf{V}_{2,0} \\
\mathbf{V}_{2,1}
\end{array}\right] \mathbf{z}
\end{aligned}
$$

where $\mathbf{z}$ is any vector of dimension 2 descibing the components of $\tau_{a}$ in $\mathbf{L}_{a}$ 's kernel and which do not create efforts.

Among these solutions, we choose via $\mathbf{z}$, the solution that follows as close as possible, the desired leg distribution determined in the balance control in equation (7) :

$$
\begin{aligned}
\mathbf{W}_{a c t, l} & =\alpha_{l} \mathbf{W}_{a c t, C O M}=\mathbf{L}_{a, l} \boldsymbol{\tau}_{a, l} \\
\mathbf{W}_{a c t, r} & =\alpha_{r} \mathbf{W}_{a c t, C O M}=\mathbf{L}_{a, r} \boldsymbol{\tau}_{a, r}
\end{aligned}
$$

Wrenches are screw-vectors (members of SE(3)) for which non-invariant norms exist. For the purpose of optimization we have to choose a metric. We choose a scaled combination of the 3D-norms of the force vector and the moment at a chosen point (COM). This metric is introduced by a weighting matrix defined as :

$$
\mathbf{H}=\operatorname{diag}\left(\left[\mathbf{H}_{\mathrm{f}}, \mathbf{H}_{\mathrm{m}}\right]\right)=\left[\begin{array}{cc}
\mu_{\mathrm{f}} \cdot \mathbf{I}_{3} & \mathbf{O}_{3} \\
\mathbf{O}_{3} & \mu_{\mathrm{m}} . \mathbf{I}_{3}
\end{array}\right]
$$

where $\mu_{\mathrm{f}}$ and $\mu_{\mathrm{m}}$ are two scalars ( $\frac{\mu_{\mathrm{m}}}{\mu_{\mathrm{f}}}$ in meters). Therefore, the criterion that minimizes the quadratic error between realized wrench and desired wrench for each leg, is :

$$
\begin{array}{r}
C=\mathbf{H}\left(\mathbf{L}_{a, l} \boldsymbol{\tau}_{a, l}-\mathbf{W}_{a c t, l}\right)^{2}+\mathbf{H}\left(\mathbf{L}_{a, r} \boldsymbol{\tau}_{a, r}-\mathbf{W}_{a c t, r}\right)^{2} \\
C=\mathbf{H}\left(\mathbf{L}_{a, l}\left(\tilde{\tau}_{a, l}+\mathbf{V}_{2,0} \mathbf{z}\right)-\mathbf{W}_{a c t, l}\right)^{2} \\
+\mathbf{H}\left(\mathbf{L}_{a, r}\left(\tilde{\tau}_{a, r}+\mathbf{V}_{2,1} \mathbf{z}\right)-\mathbf{W}_{a c t, r}\right)^{2}
\end{array}
$$

As each leg is underactuated, the simulation results show that the errors (force and moment parts) between the wrenches produced by the legs and $\mathbf{W}_{a c t, l}$ or $\mathbf{W}_{a c t, r}$ are still too high and the solution leads to a loss of sole-ground contacts. To have better results, we add interaction forces in the problem for more redundancy and to handle operatorexoskeleton interactions.

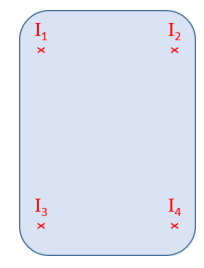

Fig. 3. Interaction points chosen between the operator and the exoskeleton at the back

d) "Optimization via the pseudoinverse matrix under leg distribution and interaction forces constraint method": The idea in this method, is to add redundancy to the problem in order to fulfill our objective (20). Therefore, we authorize $k$ interaction forces (moments are null) between the operator and the device :

$$
\begin{aligned}
& \mathbf{W}=\mathbf{W}_{a}+\mathbf{W}_{i n t} \\
& \mathbf{W}=\mathbf{L}_{a} \boldsymbol{\tau}_{a}+\sum_{i=0}^{k} \boldsymbol{\Delta}_{i} \mathbf{f}_{i} \\
& \mathbf{W}=\left[\begin{array}{lllll}
\mathbf{L}_{a, l} & \mathbf{L}_{a, r} & \boldsymbol{\Delta}_{0} & \ldots & \boldsymbol{\Delta}_{k}
\end{array}\right]\left[\begin{array}{c}
\boldsymbol{\tau}_{a, l} \\
\boldsymbol{\tau}_{a, r} \\
\mathbf{f}_{0} \\
\vdots \\
\mathbf{f}_{k}
\end{array}\right] \\
& \mathbf{W}=\mathbf{L T}
\end{aligned}
$$

with $I_{i}$ the $i^{t h}$ interaction point located at $\left[\begin{array}{lll}x_{i} & y_{i} & z_{i}\end{array}\right]^{t}$ and :

$$
\boldsymbol{\Delta}_{i} \mathbf{f}_{i}=\left[\begin{array}{ccc}
1 & 0 & 0 \\
0 & 1 & 0 \\
0 & 0 & 1 \\
0 & -z_{i} & y_{i} \\
z_{i} & 0 & -x_{i} \\
-y_{i} & x_{i} & 0
\end{array}\right]\left[\begin{array}{c}
f_{x, i} \\
f_{y, i} \\
f_{z, i}
\end{array}\right]
$$

Here, $\operatorname{dim}(\mathbf{L})=6 \times(8+3 k)$ and $\operatorname{dim}(\mathbf{T})=(8+3 k) \times 1$. Using SVD, we can write the novel set of solutions as :

$$
\mathbf{T}=\mathbf{L}^{+} \mathbf{W}_{\text {act }, C O M}+\mathbf{V}_{2} \mathbf{z}
$$

with $\operatorname{dim}\left(\mathbf{V}_{2}\right)=(8+3 k) \times(2+3 k), \operatorname{dim}(\mathbf{z})=(2+3 k) \times 1$ and $\mathbf{L}^{+}=\mathbf{L}^{t}\left(\mathbf{L} \mathbf{L}^{t}\right)^{-1}$ is the pseudoinverse matrix of $\mathbf{L}$.

We want to minimize the quadratic error between realized wrench and desired wrench that follows the desired leg distribution for each leg, and the interaction forces :

$$
C=\mathbf{H}\left(\mathbf{L}_{a, l} \boldsymbol{\tau}_{a, l}-\mathbf{W}_{a c t, l}\right)^{2}+\mathbf{H}\left(\mathbf{L}_{a, r} \boldsymbol{\tau}_{a, r}-\mathbf{W}_{a c t, r}\right)^{2}+\sum_{i=0}^{k}\left(\mathbf{f}_{i}\right)^{2}
$$


Resolving this problem means that actuators rely on interaction forces to fulfill the goal. With this criterion, we are able to quantify how much the operator has to provide in order to complete the balance control.

e) "Weighted optimization via the pseudoinverse matrix under leg distribution and interaction forces constraint method": Let's consider the criterion (36). The left leg and the right leg objectives have the same importance in the function. However intuitively, we would like to fit better the leg wrench with the smaller coefficient of leg distribution than the other. The idea is not to loose the sole-ground contact by having greater correction forces than compensation weight force when we compute joint torques. The more $\alpha$ is small, the more the associated objective is important. Let's set up this criterion :

$$
\begin{array}{r}
C=\frac{1}{\alpha_{l}} \mathbf{H}\left(\mathbf{L}_{a, l} \boldsymbol{\tau}_{a, l}-\mathbf{W}_{a c t, l}\right)^{2}+\frac{1}{\alpha_{r}} \mathbf{H}\left(\mathbf{L}_{a, r} \boldsymbol{\tau}_{a, r}-\mathbf{W}_{a c t, r}\right)^{2} \\
+\sum_{i=0}^{k}\left(\mathbf{f}_{i}\right)^{2}
\end{array}
$$

\section{Simulation RESUlTS}

We use XDE Simulator developped by CEA-LIST. Let's consider the following simulation scenario : the simulated exoskeleton is a dynamic rigid multibody system $(42 \mathrm{~kg})$ and is initially, in a stable state. We push it backwards and on the left $(50 \mathrm{~N}$ during $0,4 \mathrm{~s})$ in order to enable the correction mode by exiting ICP from the stability zone. Then, we let the system to get back into a stable state and to stabilize. Here, we observe the total wrench $\mathbf{W}_{\text {act,COM }}$ that both legs should produce. $\mathrm{x}$-axis points to the right, $\mathrm{y}$-axis forwards and $\mathrm{z}$-axis up.

In figure 4, we trace the force and moment errors between the realized right wrench from methods (a), (d) and (e) (the methods (b) and (c) are not considered because they generate too much internal efforts that lead to loose the contact between the sole and the ground) and the right wrench $\mathbf{W}_{a c t, r}$. The errors here are considered as efforts that right leg exerts on left leg. We note : methods (d) and (e) manage to realize the desired forces on fx-axis and fz-axis, however they present errors on fy-axis (up to $0,8 \mathrm{~N}$ ) ; method (a) has an important error on fx-axis when the correction mode is enabled (around $t=1,9 \mathrm{~s}$ ) ; methods (d) and (e) lead to important errors on $\mathrm{mx}$-axis $(40 \mathrm{Nm}$ and $30 \mathrm{Nm}$ ) contrary to method (a) which present no error ; on my-axis and mz-axis, the three methods present approximately the same errors.

Combining both legs in figure 5, methods (d) and (e) fit well $\mathbf{W}_{\text {act,COM }}$ (errors are zero because of equation (21)), however the method (a) presents too much error to completely realize the tasks (recovery balance and weight compensation) without creating extra forces (up to $6 \mathrm{~N}$ on fx-axis) and moments (12Nm on mz-axis). To conclude, method (e) seems to be the best to implement on the machine, because it allows the system to produce exactly the wrench we want when combining both legs and generate less internal efforts than the method (d). In figure 6, we
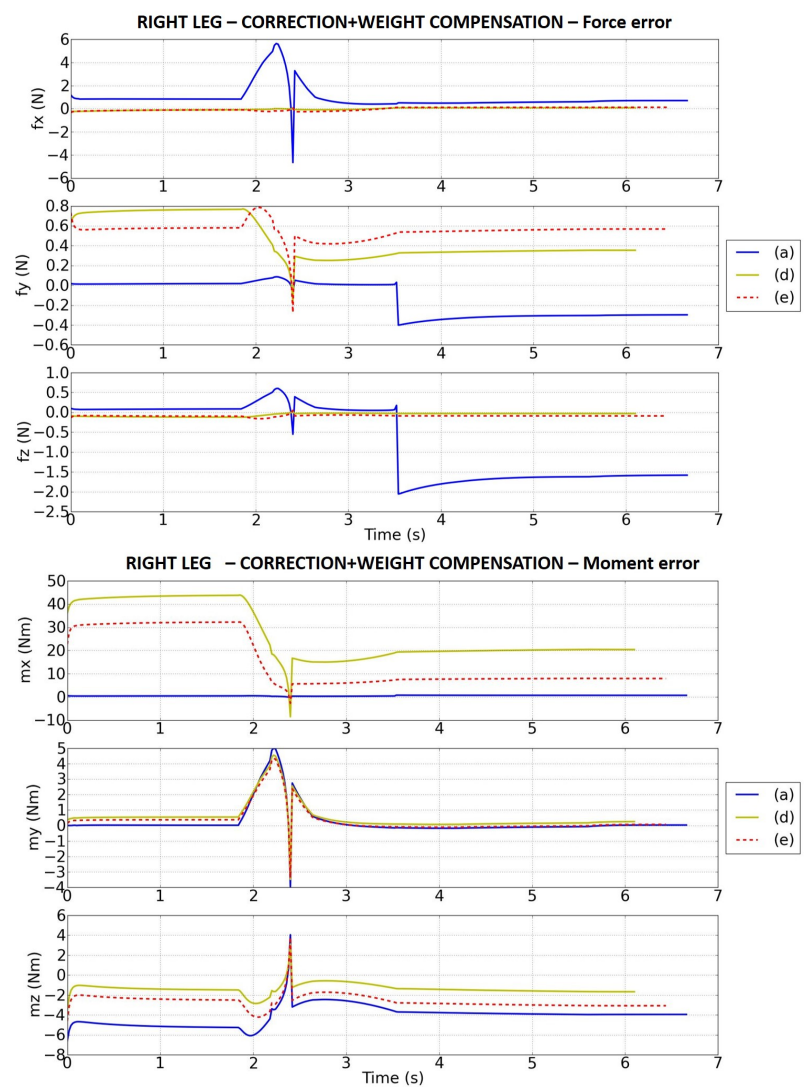

Fig. 4. Errors between $\mathbf{W}_{a c t, r}$ and the realized wrench by the right leg. Parameters : $\mu_{\mathrm{f}}=10$ and $\mu_{\mathrm{m}}=0.1$.

plot the interaction forces between the operator and the exoskeleton at points defined in figure 3. For $\mathrm{x}$-axis and $\mathrm{z}$-axis, the interaction are insignificant compared to $\mathrm{y}$-axis where we have a permanent force to compensate : $10 \mathrm{~N}$ for the maximum which represent around $1 \mathrm{~kg}$ of pressure.

All these methods lead to different results but are rather simple to implement. They also allow us to make compromises between simplicity, produced force by actuators, desired leg distribution and interaction forces. We can not conclude on which one is better without experiments but in simulations, the last one gives the best results.

\section{CONCLUSION}

We presented a balance control based on the instantaneous capture point concept and human balance recovery strategies. It is designed for an underactuated lower limbs exoskeleton where the operator is well and able and the objective was to preserve the comfort and the safety of the person inside and to maintain the balance of the entire system. Generally, all joints of an exoskeleton are not motorized in view of having machines lighter and lighter. So, dealing with a real machine implies dealing with underactuated or overactuated systems. Here, one leg of EMY-Balance exoskeleton in underactuated but combining the both legs we have an overactuated system. We proposed different methods in order to compute joint torques to control the robot, that we need to test. However, 

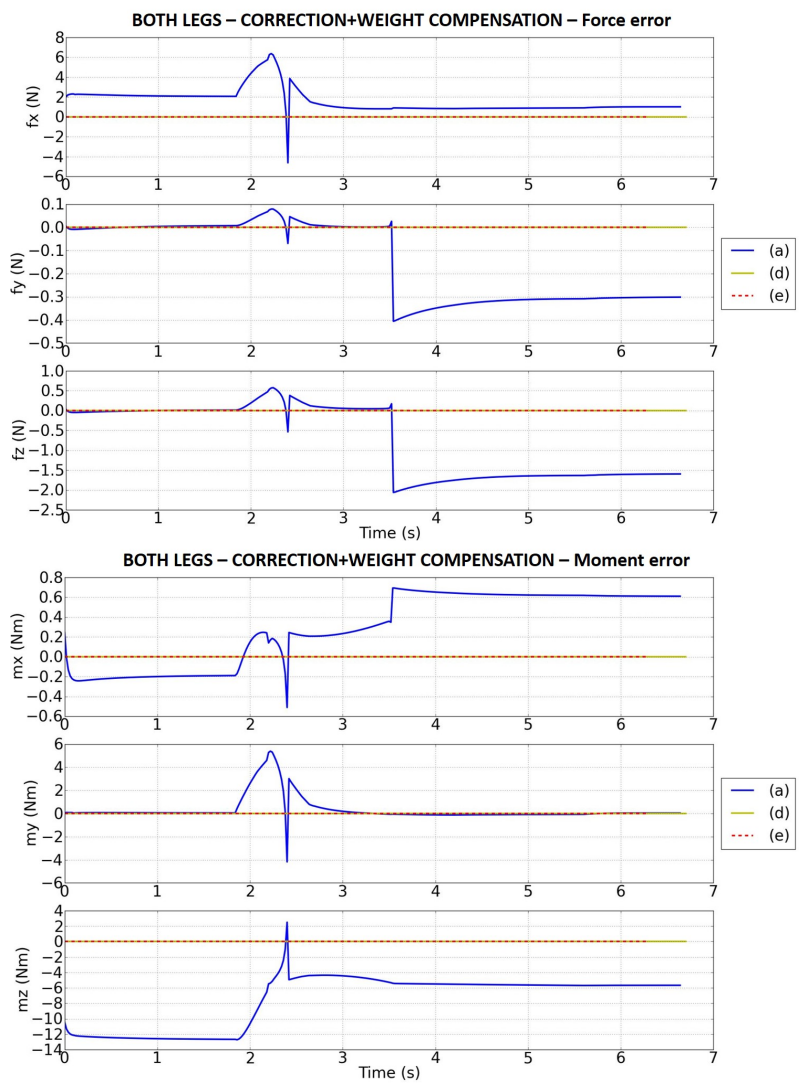

Fig. 5. Errors between $\mathbf{W}_{a c t, C O M}$ and the realized wrench by both legs. Parameters : $\mu_{\mathrm{f}}=10$ and $\mu_{\mathrm{m}}=0.1$.

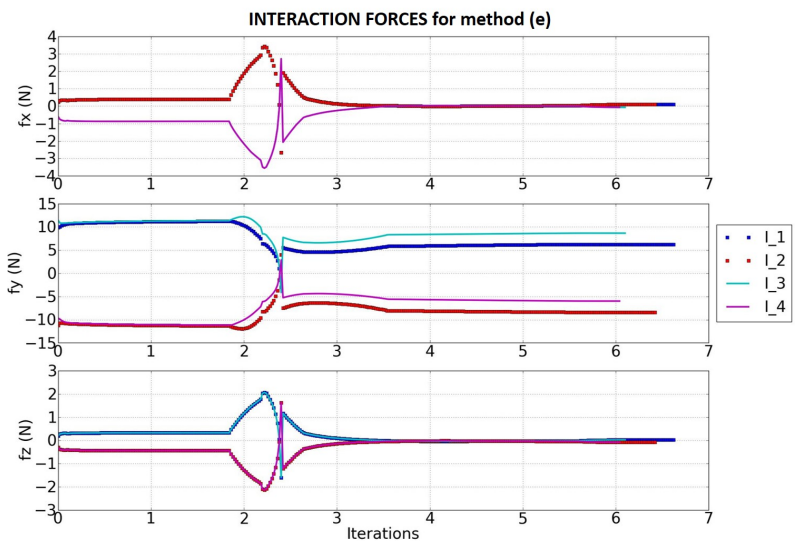

Fig. 6. Interaction forces at the 4 interaction points on the back for the method (e). In blue and in cyan, we have forces at left side interaction points (top and bottom). In red and in magenta, we have the right side's (top and bottom).

we proposed a new criteria based on leg distribution and interaction forces that gives encouraging results. We are aware that this criteria also depends on chosen interaction points between the operator and the machine. Finally, we need to evaluate how much the operator can compensate and to adjust the criteria and its weights in function of his experience.

\section{REFERENCES}

[1] A. M. Dollar and H. Herr, Lower extremity exoskeletons and active orthoses : challenges and state-of-the-art, IEEE Transactions on robotics, vol. 24, (2008)

[2] R. Bogue, Exoskeletons and robotic prosthetics : a review of recent developments, Industrial Robot : an international journal, 421-427, (2009)

[3] B. Moriniere, A. Verney, N. Abroug, P. Garrec and Y. Perrot, EMY: a dual arm exoskeleton dedicated to the evaluation of Brain Machine Interface in clinical trials, IEEE/RSJ International Conference on Intelligent Robots and Systems (IROS), pp. 5333-5338, (2015)

[4] J. L. Racine, Control of a lower limb extremity exoskeleton for human performance amplification, PhD dissertation, Univ. of California, Berkeley (2003)

[5] L. M. Mooney, E. J. Rouse and H. M. Herr, Autonomous exoskeletons reduces metabolic cost of human walking during load carriage, Journal of NeuroEngineering and Rehabilitation, (2014)

[6] S. Kajita and K. Tani, Study of dynamic biped locomotion on rugged terrain - Derivation and application of the Linear Inverted Pendulum mode, In Proc. IEEE International Conference on robotics and automation, (1991)

[7] T. Koolen, T. de Boer, J. Rebula, A. Goswami and J. Pratt, Capturability-based analysis and control of legged locomotion, Part 1 : Theory and application to three simple gait models, The international journal of robotics research, (2012)

[8] D. J. Braun and M. Goldfarb, A controller for dynamic walking in bipedal robots, In Proc. IEEE International Conference on Robotics and Automation, (2009)

[9] M. Vukobratovic, D. Hristic and Z. Stojiljkovic, Developement of active anthropomorphic exoskeletons, Medical and Biological Engineering, (1974)

[10] S. Kajita, F. Kanehiro, K. Kaneko, K. Fujiwara, K. Harada, K.Yokoi and $\mathrm{H}$. Hirukawa, Biped walking pattern generation by using preview control of zero-moment point, In Proc. IEEE International Conference on robotics and automation, (2003)

[11] P. Sardain and G. Bessonnet, Forces acting on a biped robot, center of pressure-zero moment point, IEEE Transactions on systems, man and cybernetics, vol. 34, (2004)

[12] J. Pratt, J. Carff, S. Drakunov and A. Goswami, Capture point : a step toward humanoid push recovery, In Proc. IEEE International Conference on humanoid robotics, 200-207, (2006)

[13] A. L. Hof, M. G. J. Gazendam and W. E. Sinke, The condition for dynamic stability, Journal of biomechanics, vol. 38, 1-8, (2004)

[14] D. A. Winter, Human balance and posture control during standing and walking, Gait \& Posture, vol. 3, 193-214, (1995)

[15] F. B. Horak and L. M. Nashner, Central programming of postural movements : adaptation to altered support surface configurations, Journal of Neurophysiol, vol. 55, no. 6, 1369-1381, (1986)

[16] A. L. Hof, The equations of motion for a standing human reveal three mechanisms for balance, Journal of biomechanics, vol. 40, 451-457, (2005)

[17] A. L. Hof, The 'extrapoled center of mass' concept suggests a simple control of balance in walking, Human movement science, vol. 27, $112-125,(2007)$

[18] V. Huynh, C. Bidard and C. Chevallereau, Balance control for an active leg exoskeleton based on human balance strategies, International workshop on medical and service robots, (MESROB 2016)

[19] H. Herr and M. Popovic, Angular momentum in human walking, The journal of experimental biology, vol. 211, (2008)

[20] L. Kaul, C.Mandery, D. Busch, N. Vahrenkamp and T. Asfour, Experimental analysis of human push recovery by stepping, KoiroiBot, (2015)

[21] P. Garrec and F. Kfoury, Misalignment-tolerant cable actuator, Patent WO2014128178 A1, (2014)

[22] J. Englsberger, C. Ott, M. A. Roa, A. Albu-Schffer and G. Hirzinger, Bipedal walking control based on capture point dynamics, In Proc. IEEE International Conference on intelligent robots and systems, (2011)

[23] J. Pratt, T. Koolen, T. de Boer, J. Rebula, S. Cotton, J. Carff, M. Johnson and P. Neuhaus, Capturability-based analysis and control of legged locomotion, Part 2 : Application to M2V2, a lower-body humanoid, The international journal of robotics research, (2012)

[24] J. Pratt and G. Pratt, Intuitive control of planar bipedal walking robot, In Proc. IEEE International Conference on Robotics and Automation, (1998) 